\title{
Efeitos do sistema de plantio e da consorciação soja-milho sobre artrópodes capturados no solo(1)
}

\author{
Francisco Jorge Cividanes ${ }^{(2)}$
}

\begin{abstract}
Resumo - O objetivo deste estudo foi avaliar os efeitos do plantio direto e da consorciação soja [Glycine $\max (\mathrm{L}$.) Merrill] e milho (Zea mays L.) sobre artrópodes que vivem no solo. Os tratamentos constituíram um fatorial 3 x 2 (monocultura de soja, monocultura de milho, consorciação soja-milho x plantio direto, plantio convencional) em blocos casualizados com quatro repetições; as amostragens foram efetuadas com armadilhas de solo, durante 1996 a 1998. Resultados estatisticamente significativos em pelo menos uma safra indicaram que o plantio direto foi favorável a predadores, como formigas (Brachymyrmex sp., Camponotus spp. e Ectatomma sp.), carabídeos (Metius sp.) e aranhas (Lycosidae). A consorciação soja-milho aumentou o número de Acrididae, enquanto a presença do milho favoreceu a ocorrência de Dermaptera em sistemas de consorciação soja-milho.
\end{abstract}

Termos para indexação: Glycine max, Zea mays, plantio direto, insetos, predadores.

\section{Effects of tillage systems and intercropping of soybean and corn on soil-inhabiting arthropods}

\begin{abstract}
The objective of this study was to evaluate the effects of no-tillage and strip intercropping of soybean [Glycine $\max$ (L.) Merrill] and corn (Zea mays L.) on the abundance of soil-inhabiting arthropods. The plots were arranged in a randomized complete block design for a 3 by 2 factorial experiment (soybean monoculture, corn monoculture, strip intercropping of soybean-corn x no-tillage, conventional tillage). Each treatment was replicated four times. The arthropods were sampled by pitfall traps during 1996 - 1998. Statistically significant results from at least one season indicated that notillage was favorable to predators like ants (Brachymyrmex sp., Camponotus spp. and Ectatomma sp.), carabids (Metius sp.) and Araneae (Lycosidae). Strip intercropping increased the number of Acrididae, while the presence of corn was favorable to the occurrence of Dermaptera in strip intercropping of soybean and corn.
\end{abstract}

Index terms: Glycine max, Zea mays, direct sowing, insects, predators.

\section{Introdução}

O plantio direto e a consorciação de culturas são considerados fatores de diversificação do agroecossistema, por aumentarem a diversidade de estruturas vegetais e de espécies de plantas (Emden \& Williams, 1974). Essa diversidade pode contribuir para a ocorrência de maior abundância e diversidade de inimigos naturais (Symondson et al., 1996; Andersen, 1999) e de menor número de insetos fitófagos (Garcia \& Altieri, 1992).

\footnotetext{
(1) Aceito para publicação em 5 de dezembro de 2000 . Financiado pela Fapesp.

(2) Universidade Estadual Paulista, Faculdade de Ciências Agrárias e Veterinárias, Dep. de Fitossanidade, Via de Acesso Prof. Paulo Donato Castellane, s/n, CEP 14870-000 Jaboticabal, SP. Bolsista do CNPq. E-mail: fjcivida@fcav.unesp.br
}

No entanto, o efeito da consorciação de culturas sobre artrópodes ainda constitui um assunto controverso (Letourneau, 1990). Entre as prováveis explicações pode-se citar a enorme complexidade da interação planta-artrópode em agroecossistemas diversificados, pois a variação da densidade de artrópodes nesses agroecossistemas depende de muitos fatores, como: densidade de plantio, culturas envolvidas, adaptabilidade do predador à cultura, densidade populacional das presas, especificidade do predador, disponibilidade de outras fontes de alimento, umidade do solo e microclima das culturas (Letourneau, 1990; Stinner \& House, 1990; Booij \& Noorlander, 1992; Cárcamo \& Spence, 1994; Clark et al., 1997; French et al., 1998).

Os artrópodes predadores encontrados no solo de culturas agrícolas pertencem, basicamente, aos gru- 
pos das aranhas, carabídeos, formigas e estafilinídeos (Stinner \& House, 1990), e, como são importantes para o controle de pragas, faz-se necessário conhecer a resposta deles às diferentes práticas de manejo das culturas, para que se possam determinar opções para aumentar a densidade desses predadores nos sistemas de produção agrícola (Andow, 1992; Booij \& Noorlander, 1992; Clark et al., 1997).

O objetivo deste estudo foi avaliar a influência do plantio direto e da consorciação soja-milho sobre artrópodes que vivem no solo.

\section{Material e Métodos}

$\mathrm{O}$ estudo foi realizado na área experimental do Campus da FCAV-Unesp, Jaboticabal, SP, durante o período de dezembro de 1996 a setembro de 1998. A área do experimento era de 1,6 ha $(26 \times 620 \mathrm{~m})$, e os tratamentos constituíram um fatorial 3 x 2 (monocultura de soja, monocultura de milho, soja-milho consorciados x plantio direto, plantio convencional), em blocos casualizados, com quatro repetições. As parcelas ficaram separadas entre si por $9 \mathrm{~m}$, sendo assim constituídas: a) soja, 40 linhas de plantas, a espaços de $0,5 \mathrm{~m}$ entre linhas, e dimensões de 16 x $20 \mathrm{~m}$; b) milho, 20 linhas de plantas com espaços, entre si, de $0,90 \mathrm{~m}$ na linha, com dimensões de $16 \times 18 \mathrm{~m}$; c) consorciadas, três faixas de milho de cinco linhas de plantas, cada, alternadas com duas faixas de dez linhas de soja, cada, tendo dimensões de $16 \times 23,5 \mathrm{~m}$.

Durante a safra 1995/96, a área experimental foi cultivada com soja, no sistema convencional; a seguir plantou-se sorgo no sistema de plantio direto. Após a colheita deste, a área foi deixada em pousio. Nas duas safras estudadas, o preparo da área foi iniciado com a aplicação do herbicida glyphosate (Roundap Glifosato - dose de 2,0 L/ha), sendo feito, a seguir, o corte do mato seco, com um triturador de palha. Nas parcelas de plantio convencional, o preparo do solo foi feito por meio de uma aração (arado de disco) e uma gradagem (grade de discos pesada). A semeadura, em todas as parcelas experimentais, foi efetuada em 28/11/96 e 9/12/97, utilizando-se a cultivar BR-16 de soja e os híbridos de milho 'Pionner 3041' (safra 1996/97) e 'Cargill C-701' (safra 1997/98). Em seguida, aplicou-se o herbicida metolachlor (Dual $960 \mathrm{CE}$ - dose 2,0 L/ha) sobre a área semeada, e paraquat (Gramoxone 200 - dose de $1,0 \mathrm{~L} / \mathrm{ha}$ ), na área não semeada. A adubação foi feita com $330 \mathrm{~kg} /$ ha da fórmula 2-20-18 (NPK). Durante as safras, as parcelas foram mantidas no limpo, por meio de capinas periódicas no interior delas, enquanto ao redor delas as plantas daninhas foram cortadas com um triturador de palha.
Não foi aplicado inseticida na área experimental durante o período do estudo.

Os artrópodes predadores foram amostrados por meio de armadilhas de solo (alçapão), colocadas em número de duas, separadas um metro entre si, no centro das parcelas (Tonhasca Junior, 1993; Cárcamo \& Spence, 1994). Como armadilhas foram usados copos de plástico com $8 \mathrm{~cm}$ de diâmetro e $14 \mathrm{~cm}$ de altura, que recebiam cerca de $150 \mathrm{~mL}$ de água com algumas gotas de detergente neutro, permanecendo 48 horas no campo (Tonhasca Junior, 1993). A freqüência de amostragem dependeu das condições de precipitação, tendo sido feita, em média, a cada duas semanas durante a safra, e a cada três semanas na entressafra. Nos períodos de entressafra, a área experimental ficou em pousio, e as parcelas permaneceram marcadas com estacas para receberem o mesmo manejo do solo e cultura da safra anterior. Os resultados foram submetidos a análise da variância, e as médias, comparadas pelo teste de Tukey.

\section{Resultados e Discussão}

Foram capturados seis grupos de artrópodes predadores (Tabela 1). O grupo das formigas foi o mais numeroso, vindo, a seguir, em ordem decrescente, Araneae, Carabidae, Dermaptera, Staphylinidae e Cicindelidae, e foram identificados nove gêneros/espécies de formigas, doze famílias e nove gêneros/espécies de aranhas, e oito gêneros/espécies de carabídeos. Ressalte-se, que, ante a dificuldade de identificação, várias espécies de aranhas e carabídeos não foram classificadas a nível de família e espécie, respectivamente. Com relação a insetos fitófagos, foram capturadas várias espécies de ortópteros das famílias Acrididae e Gryllidae. Foram mais abundantes indivíduos pertencentes a esta última família.

No geral, a densidade populacional dos artrópodes diferiu de uma safra para outra. De acordo com Tonhasca Junior \& Stinner (1991) e Tonhasca Junior (1993), podem ocorrer variações no número desses organismos presentes em culturas, por causa das diferenças na precipitação pluvial registrada em cada safra. De fato, nos meses iniciais das safras (dezembro a janeiro) a precipitação pluvial na safra 1996/97 foi 2,2 vezes maior do que na safra 1997/98. Naquela safra ocorreu maior número de Dermaptera e Carabidae, destacando-se, nesta família, Abaris sp., Callida scutellaris, Colliuris sp. e Galerita sp. Tais resultados podem evidenciar que a maior precipitação registrada na safra 1996/97 foi 
Tabela 1. Total de artrópodes predadores e fitófagos capturados com armadilhas de solo em safras e entressafras de soja e milho cultivados em sistemas de plantio direto, convencional e consorciação. Jaboticabal, SP.

\begin{tabular}{|c|c|c|c|c|}
\hline Categorias taxonômicas & Safra 96/97 & Entressafra/97 & Safra 97/98 & Entressafra/98 \\
\hline \multicolumn{5}{|l|}{ Predadores } \\
\hline \multicolumn{5}{|l|}{ Formicidae } \\
\hline Pheidole spp. & 4.030 & 8.202 & 6.050 & 4.896 \\
\hline Dorymyrmex sp. & 2.146 & 6.900 & 1.941 & 1.756 \\
\hline Crematogaster sp. & 278 & 1.189 & 487 & 639 \\
\hline Solenopsis spp. & 172 & 342 & 1.110 & 439 \\
\hline Brachymyrmex sp. & 12 & 163 & 63 & 285 \\
\hline Gnamptogenys sp. & 208 & 8 & 276 & 40 \\
\hline Ectatomma sp. & 64 & 29 & 273 & 60 \\
\hline Camponotus spp. & 38 & 69 & 136 & 162 \\
\hline Odontomachus sp. & 20 & 3 & 19 & 6 \\
\hline \multicolumn{5}{|l|}{ Carabidae } \\
\hline Abaris sp. & 49 & 57 & 11 & 1 \\
\hline Metius sp. & 52 & 8 & 43 & 12 \\
\hline Callida scutellaris & 34 & 21 & 2 & 0 \\
\hline Lebia spp. & 7 & 1 & 14 & 3 \\
\hline Colliuris sp. & 13 & 0 & 0 & 0 \\
\hline Galerita sp. & 12 & 1 & 0 & 0 \\
\hline Scarites sp. & 3 & 0 & 3 & 0 \\
\hline Calosoma granulatum & 3 & 0 & 0 & 0 \\
\hline Outros & 40 & 10 & 6 & 0 \\
\hline Dermaptera & 158 & 4 & 20 & 1 \\
\hline Staphylinidae & 22 & 16 & 10 & 3 \\
\hline Cicindelidae & 16 & 0 & 12 & 0 \\
\hline \multicolumn{5}{|l|}{ Araneae } \\
\hline \multicolumn{5}{|l|}{ Lycosidae } \\
\hline Lycosa erythrognatha & 2 & 4 & 1 & 6 \\
\hline Outras & 57 & 106 & 93 & 47 \\
\hline Linyphiidae & 12 & 18 & 71 & 73 \\
\hline \multicolumn{5}{|l|}{ Salticidae } \\
\hline Oxyopes salticus & 5 & 8 & 8 & 1 \\
\hline Ailluticus sp. & 1 & 0 & 0 & 0 \\
\hline Outras & 7 & 40 & 18 & 31 \\
\hline \multicolumn{5}{|l|}{ Gnaphosidae } \\
\hline Apopyllus silvestrii & 11 & 6 & 7 & 12 \\
\hline Camillina pulcher & 21 & 4 & 10 & 7 \\
\hline Outras & 6 & 2 & 3 & 2 \\
\hline \multicolumn{5}{|l|}{ Titanoecidae } \\
\hline Goeldia sp. & 4 & 0 & 7 & 2 \\
\hline Araneidae & 6 & 3 & 0 & 0 \\
\hline \multicolumn{5}{|l|}{ Corinnidae } \\
\hline Falconita sp. & 1 & 0 & 4 & 3 \\
\hline Orthobula sp. & 1 & 0 & 0 & 0 \\
\hline Lyssomaridae & 0 & 0 & 2 & 0 \\
\hline Theridiidae & 2 & 0 & 0 & 0 \\
\hline Clubionidae & 1 & 0 & 0 & 0 \\
\hline Haniidae & 1 & 0 & 0 & 0 \\
\hline \multicolumn{5}{|l|}{ Tetragnathidae } \\
\hline Leucage sp. & 1 & 0 & 0 & 0 \\
\hline Outras famílias & 70 & 133 & 111 & 65 \\
\hline \multicolumn{5}{|l|}{ Fitófagos } \\
\hline Acrididae & 234 & 894 & 310 & 515 \\
\hline Gryllidae & 2.418 & 450 & 485 & 259 \\
\hline
\end{tabular}


favorável à ocorrência destes insetos em alta densidade populacional, pois, segundo Watmough \& Kfir (1995) e French et al. (1998), a espécie Dorylus helvolus (Formicidae) e várias outras espécies de Carabidae e Dermaptera foram mais abundantes em períodos de maior precipitação pluvial. Algumas espécies de Formicidae, como Solenopsis spp., Ectatomma sp. e Camponotus spp., foram mais numerosas na safra 1997/98, e observou-se pouca variação no número de aranhas capturadas nas duas safras (Tabela 1). Como nessa safra a precipitação foi menor do que na safra anterior, pode-se inferir que as referidas espécies de formi- gas podem ter sido favorecidas pela menor precipitação, enquanto as aranhas sofreram pouca influência desse fator meteorológico.

Vários trabalhos mostraram que a densidade de carabídeos, formigas e aranhas aumenta com práticas agrícolas que reduzem a movimentação do solo (Stinner \& House, 1990; Clark et al., 1997; Marasas et al., 1997). O presente estudo confirmou estas observações, pois aranhas, com destaque para Lycosidae, carabídeos, principalmente Metius sp., e formigas Camponotus spp., Brachymyrmex sp. e Ectatomma sp., ocorreram em maior número no plantio direto (Tabelas 2 e 3). A maior densidade des-

Tabela 2. Número médio de artrópodes predadores e fitófagos amostrados com armadilhas de solo na safra 1996/97. Jaboticabal, $\mathrm{SP}^{(1)}$.

\begin{tabular}{|c|c|c|c|c|c|c|c|}
\hline \multirow[t]{2}{*}{ Categorias taxonômicas } & \multicolumn{2}{|c|}{ Manejo do solo } & \multicolumn{3}{|c|}{ Culturas } & \multirow[t]{2}{*}{ Erro-padrão } & \multirow[t]{2}{*}{ CV (\%) } \\
\hline & Direto & Convencional & Soja & Milho & Soja $\mathrm{x}$ milho & & \\
\hline \multicolumn{8}{|l|}{ Predadores } \\
\hline \multicolumn{8}{|l|}{ Formicidae } \\
\hline Brachymyrmex sp. & $0,1 \mathrm{a}$ & $0,05 \mathrm{a}$ & $0,05 \mathrm{a}$ & $0,03 \mathrm{a}$ & $0,1 \mathrm{a}$ & 0,07 & 177,2 \\
\hline Camponotus spp. & $0,3 \mathrm{a}$ & $0,08 \mathrm{~b}$ & $0,1 \mathrm{a}$ & $0,3 \mathrm{a}$ & $0,2 \mathrm{a}$ & 0,12 & 54,1 \\
\hline Crematogaster sp. & $2,8 \mathrm{a}$ & $0,1 \mathrm{a}$ & $3,6 a$ & $0,2 \mathrm{a}$ & $0,5 \mathrm{a}$ & 2,28 & 115,0 \\
\hline Dorymyrmex sp. & $13,3 \mathrm{a}$ & $9,1 \mathrm{a}$ & $9,1 \mathrm{a}$ & $9,6 \mathrm{a}$ & $14,8 \mathrm{a}$ & 2,90 & 50,3 \\
\hline Ectatomma sp. & $0,4 \mathrm{a}$ & $0,3 \mathrm{a}$ & $0,4 \mathrm{a}$ & $0,3 \mathrm{a}$ & $0,4 a$ & 0,13 & 78,2 \\
\hline Gnamptogenys sp. & $1,2 \mathrm{a}$ & $1,1 \mathrm{a}$ & $1,2 \mathrm{a}$ & $1,0 \mathrm{a}$ & $1,1 \mathrm{a}$ & 0,40 & 72,3 \\
\hline Odontomachus sp. & $0,2 \mathrm{a}$ & $0,05 \mathrm{a}$ & $0,1 \mathrm{a}$ & $0,1 \mathrm{a}$ & $0,1 \mathrm{a}$ & 0,07 & 130,7 \\
\hline Pheidole spp. & $22,3 \mathrm{a}$ & $19,7 \mathrm{a}$ & $21,7 \mathrm{a}$ & $18,1 \mathrm{a}$ & $23,2 \mathrm{a}$ & 3,83 & 34,5 \\
\hline Solenopsis spp. & $1,1 \mathrm{a}$ & $0,7 \mathrm{a}$ & $1,3 \mathrm{a}$ & $0,7 \mathrm{a}$ & $0,8 \mathrm{a}$ & 0,82 & 183,3 \\
\hline Total & $41,5 \mathrm{a}$ & $31,2 \mathrm{~b}$ & $37,5 \mathrm{a}$ & $30,3 a$ & $41,2 \mathrm{a}$ & 5,51 & 30,4 \\
\hline \multicolumn{8}{|l|}{ Carabidae } \\
\hline Abaris sp. & $0,3 \mathrm{a}$ & $0,2 \mathrm{a}$ & $0,4 \mathrm{a}$ & $0,2 \mathrm{a}$ & $0,2 \mathrm{a}$ & 0,18 & 137,7 \\
\hline Callida scutellaris & $0,3 \mathrm{a}$ & $0,1 \mathrm{a}$ & $0,1 \mathrm{a}$ & $0,3 a$ & $0,2 \mathrm{a}$ & 0,11 & 117,1 \\
\hline Metius sp. & $0,4 \mathrm{a}$ & $0,2 b$ & $0,3 \mathrm{a}$ & $0,3 \mathrm{a}$ & $0,2 \mathrm{a}$ & 0,07 & 53,1 \\
\hline Total & $1,7 \mathrm{a}$ & $1,0 \mathrm{~b}$ & $1,5 \mathrm{a}$ & $1,2 \mathrm{a}$ & $1,4 \mathrm{a}$ & 0,34 & 56,8 \\
\hline Cicindelidae & $0,1 \mathrm{a}$ & $0,1 \mathrm{a}$ & $0,0 \mathrm{a}$ & $0,2 \mathrm{a}$ & $0,1 \mathrm{a}$ & 0,10 & 139,8 \\
\hline Staphylinidae & $0,1 \mathrm{a}$ & $0,1 \mathrm{a}$ & $0,1 \mathrm{a}$ & $0,2 \mathrm{a}$ & $0,1 \mathrm{a}$ & 0,12 & 148,2 \\
\hline Dermaptera & $0,8 \mathrm{a}$ & $0,9 \mathrm{a}$ & $0,3 b$ & $0,9 \mathrm{a}$ & $1,3 a$ & 0,22 & 50,3 \\
\hline \multicolumn{8}{|l|}{ Araneae } \\
\hline Lycosidae & $0,4 \mathrm{a}$ & $0,2 \mathrm{~b}$ & $0,4 \mathrm{a}$ & $0,2 \mathrm{a}$ & $0,3 \mathrm{a}$ & 0,09 & 65,4 \\
\hline Total & $1,4 \mathrm{a}$ & $0,8 \mathrm{~b}$ & $1,1 \mathrm{a}$ & $0,9 \mathrm{a}$ & $1,3 \mathrm{a}$ & 0,21 & 42,9 \\
\hline \multicolumn{8}{|l|}{ Fitófagos } \\
\hline Acrididae & $1,3 \mathrm{a}$ & $1,2 \mathrm{a}$ & $1,3 \mathrm{ab}$ & $0,7 \mathrm{~b}$ & $1,6 \mathrm{a}$ & 0,30 & 49,9 \\
\hline Gryllidae & $10,1 \mathrm{~b}$ & $15,1 \mathrm{a}$ & $9,6 \mathrm{a}$ & $12,2 \mathrm{a}$ & $15,9 \mathrm{a}$ & 2,54 & 39,4 \\
\hline
\end{tabular}

${ }^{(1)}$ Médias seguidas da mesma letra, na horizontal, entre sistemas de plantio e entre culturas, não diferem entre si pelo teste de Tukey a 5\% de probabilidade; não foram significativas as interações entre manejo de solo e culturas. 
ses artrópodes contribui para o controle de pragas agrícolas, o que pode reduzir a necessidade de aplicação de inseticidas e aumentar os lucros dos agricultores (Hook, 1971; Brust \& House, 1990; Brust, 1991; Clark et al., 1994; Sunderland et al., 1995; French et al., 1998).

Insetos da ordem Dermaptera, principalmente Doru sp., foram observados apenas durante a safra e entressafra de 1996/97, e ocorreram em número significativamente maior na monocultura do milho e nas parcelas de consorciação soja-milho, durante a referida safra (Tabelas 2 e 4). Apesar de as diferenças entre as densidades populacionais dos outros predadores capturados nos dois tipos de plantio não terem sido significativas, o total de aranhas tendeu a ocorrer em maior número nas parcelas de monocultura de soja e na consorciação soja-milho durante as duas safras estudadas (Tabelas 2 e 3); o mesmo verificou-se com o total de formigas na safra 1996/97 (Tabela 2). Na entressafra 1997, as formigas foram significativamente mais abundantes nas parcelas destinadas ao monocultivo de soja e consorciação soja-milho (Tabela 4), enquanto aranhas da família Linyphiidae apresentaram-se em número significativamente maior nas parcelas utilizadas para o consórcio soja-milho na entressafra de 1998 (Tabela 5). Es-

Tabela 3. Número médio de artrópodes predadores e fitófagos amostrados com armadilhas de solo na safra 1997/98. Jaboticabal, $\mathrm{SP}^{(1)}$.

\begin{tabular}{|c|c|c|c|c|c|c|c|}
\hline \multirow[t]{2}{*}{ Categorias taxonômicas } & \multicolumn{2}{|c|}{ Manejo do solo } & \multicolumn{3}{|c|}{ Culturas } & \multirow[t]{2}{*}{ Erro-padrão } & \multirow[t]{2}{*}{$\mathrm{CV}(\%)$} \\
\hline & Direto & Convencional & Soja & Milho & Soja $\mathrm{x}$ milho & & \\
\hline \multicolumn{8}{|l|}{ Predadores } \\
\hline \multicolumn{8}{|l|}{ Formicidae } \\
\hline Brachymyrmex sp. & $0,6 \mathrm{a}$ & $0,02 b$ & $0,6 \mathrm{a}$ & $0,2 \mathrm{a}$ & $0,2 \mathrm{a}$ & 0,25 & 150,7 \\
\hline Camponotus spp. & $1,2 \mathrm{a}$ & $0,2 b$ & $0,5 \mathrm{a}$ & $0,7 \mathrm{a}$ & $0,9 \mathrm{a}$ & 0,25 & 71,3 \\
\hline Crematogaster sp. & $5,1 \mathrm{a}$ & $0,2 \mathrm{a}$ & $0,2 \mathrm{a}$ & $7,2 \mathrm{a}$ & $0,3 \mathrm{a}$ & 5,14 & 280,1 \\
\hline Dorymyrmex sp. & $9,9 a$ & $10,3 \mathrm{a}$ & $4,2 \mathrm{a}$ & $12,7 \mathrm{a}$ & $13,4 \mathrm{a}$ & 7,42 & 127,2 \\
\hline Ectatomma sp. & $2,2 \mathrm{a}$ & $0,6 b$ & $1,4 \mathrm{a}$ & $1,4 \mathrm{a}$ & $1,4 \mathrm{a}$ & 0,49 & 69,6 \\
\hline Gnamptogenys sp. & $1,3 \mathrm{a}$ & $1,6 \mathrm{a}$ & $1,7 \mathrm{a}$ & $1,3 \mathrm{a}$ & $1,4 \mathrm{a}$ & 0,36 & 50,6 \\
\hline Odontomachus sp. & $0,1 \mathrm{a}$ & $0,1 \mathrm{a}$ & $0,1 \mathrm{a}$ & $0,03 \mathrm{a}$ & $0,2 \mathrm{a}$ & 0,09 & 175,1 \\
\hline Pheidole spp. & $34,9 \mathrm{a}$ & $28,1 \mathrm{a}$ & $24,6 \mathrm{a}$ & $34,4 \mathrm{a}$ & $35,5 \mathrm{a}$ & 12,69 & 83,2 \\
\hline Solenopsis spp. & $7,5 \mathrm{a}$ & $4,1 \mathrm{a}$ & $9,1 \mathrm{a}$ & $3,0 \mathrm{a}$ & $5,3 \mathrm{a}$ & 6,40 & 121,4 \\
\hline Total & $62,8 \mathrm{a}$ & $45,1 \mathrm{a}$ & $42,3 \mathrm{a}$ & $61,0 \mathrm{a}$ & $58,5 \mathrm{a}$ & 14,86 & 55,1 \\
\hline \multicolumn{8}{|l|}{ Carabidae } \\
\hline Metius sp. & $0,4 \mathrm{a}$ & $0,1 \mathrm{~b}$ & $0,3 \mathrm{a}$ & $0,2 \mathrm{a}$ & $0,2 \mathrm{a}$ & 0,09 & 83,8 \\
\hline Total & $0,5 \mathrm{a}$ & $0,3 b$ & $0,5 \mathrm{a}$ & $0,3 \mathrm{a}$ & $0,3 \mathrm{a}$ & 0,13 & 68,0 \\
\hline \multicolumn{8}{|l|}{ Araneae } \\
\hline Lycosidae & $0,7 \mathrm{a}$ & $0,3 b$ & $0,5 \mathrm{a}$ & $0,6 \mathrm{a}$ & $0,5 \mathrm{a}$ & 0,10 & 43,5 \\
\hline Linyphiidae & $0,4 a$ & $0,3 \mathrm{a}$ & $0,3 \mathrm{a}$ & $0,4 a$ & $0,4 a$ & 0,12 & 64,8 \\
\hline Salticidae & $0,2 \mathrm{a}$ & $0,1 \mathrm{a}$ & $0,1 \mathrm{a}$ & $0,1 \mathrm{a}$ & $0,1 \mathrm{a}$ & 0,06 & 85,8 \\
\hline Total & $2,2 \mathrm{a}$ & $1,3 b$ & $1,6 \mathrm{a}$ & $1,5 \mathrm{a}$ & $1,8 \mathrm{a}$ & 0,24 & 29,1 \\
\hline \multicolumn{8}{|l|}{ Fitófagos } \\
\hline Acrididae & $1,8 \mathrm{a}$ & $1,4 \mathrm{a}$ & $1,7 \mathrm{a}$ & $1,5 \mathrm{a}$ & $1,6 \mathrm{a}$ & 0,27 & 33,9 \\
\hline Gryllidae & $1,9 b$ & $3,1 \mathrm{a}$ & $2,4 \mathrm{a}$ & $2,9 \mathrm{a}$ & $2,3 \mathrm{a}$ & 0,44 & 35,2 \\
\hline
\end{tabular}

${ }^{(1)}$ Médias seguidas da mesma letra, na horizontal, entre sistemas de plantio e entre culturas, não diferem entre si pelo teste de Tukey a $5 \%$ de probabilidade; não foram significativas as interações entre manejo do solo e culturas. 
ses resultados em parte concordam com os encontrados por outros autores, que, estudando a consorciação soja-milho (Tonhasca Junior \& Stinner, 1991; Tonhasca Junior, 1993) e milho-caupi (Skovgard \& Pats, 1996) não constataram influência desse tipo de plantio sobre predadores que vivem no solo.

Com relação a insetos fitófagos, várias espécies da ordem Orthoptera foram capturadas. As pertencentes à família Gryllidae ocorreram em número significativamente menor nas condições de plantio direto, nas duas safras estudadas, enquanto ortópteros da família Acrididae não sofreram influência do manejo de solo (Tabelas 2 a 5). Esses resultados diferem dos obtidos por Tonhasca Junior (1994), que encontrou maior abundância de Acrididae em sistemas de plantio direto de soja e milho do que nos de plantio convencional dessas culturas. A menor densidade de Gryllidae verificada nos sistemas de plantio direto pode ter sido causada pela predação efetuada por aranhas, pois Hook (1971) ressaltou que a ação de aranhas, principalmente Lycosa spp., causou a mortalidade de $66 \%$ da população de grilos

Tabela 4. Número médio de artrópodes predadores e fitófagos amostrados com armadilhas de solo na entressafra/ 1997. Jaboticabal, $\mathrm{SP}^{(1)}$.

\begin{tabular}{|c|c|c|c|c|c|c|c|}
\hline \multirow[t]{2}{*}{ Categorias taxonômicas } & \multicolumn{2}{|c|}{ Manejo do solo } & \multicolumn{3}{|c|}{ Cultura utilizada na parcela } & \multirow{2}{*}{ Erro-padrão } & \multirow[t]{2}{*}{$\mathrm{CV}(\%)$} \\
\hline & Direto & Convencional & Soja & Milho & Soja $\mathrm{x}$ milho & & \\
\hline \multicolumn{8}{|l|}{ Predadores } \\
\hline \multicolumn{8}{|l|}{ Formicidae } \\
\hline Brachymyrmex sp. & $2,0 \mathrm{a}$ & $0,4 \mathrm{~b}$ & $1,4 \mathrm{a}$ & $0,4 \mathrm{a}$ & $1,7 \mathrm{a}$ & 0,83 & 142,8 \\
\hline Camponotus spp. & $0,6 \mathrm{a}$ & $0,5 \mathrm{a}$ & $0,6 \mathrm{a}$ & $0,4 \mathrm{a}$ & $0,7 \mathrm{a}$ & 0,23 & 82,6 \\
\hline Crematogaster sp. & $14,9 \mathrm{a}$ & $0,8 b$ & $14,9 \mathrm{a}$ & $4,2 \mathrm{a}$ & $4,6 \mathrm{a}$ & 6,52 & 165,7 \\
\hline Dorymyrmex sp. & $33,9 \mathrm{a}$ & $24,7 \mathrm{a}$ & $33,2 \mathrm{a}$ & $13,5 \mathrm{a}$ & $41,2 \mathrm{a}$ & 10,54 & 74,4 \\
\hline Ectatomma sp. & $0,4 \mathrm{a}$ & $0,2 \mathrm{~b}$ & $0,3 \mathrm{a}$ & $0,3 a$ & $0,2 \mathrm{a}$ & 0,05 & 36,9 \\
\hline Gnamptogenys sp. & $0,1 \mathrm{a}$ & $0,1 \mathrm{a}$ & $0,1 \mathrm{a}$ & $0,1 \mathrm{a}$ & $0,1 \mathrm{a}$ & 0,07 & 187,2 \\
\hline Odontomachus sp. & $0,02 \mathrm{a}$ & $0,01 \mathrm{a}$ & $0,02 \mathrm{a}$ & $0,01 \mathrm{a}$ & $0,00 \mathrm{a}$ & 0,02 & 101,1 \\
\hline Pheidole spp. & $42,4 \mathrm{a}$ & $44,0 \mathrm{a}$ & $41,0 \mathrm{a}$ & $39,2 \mathrm{a}$ & $49,4 \mathrm{a}$ & 8,75 & 36,1 \\
\hline Solenopsis spp. & $1,2 \mathrm{a}$ & $2,0 \mathrm{a}$ & $2,7 \mathrm{a}$ & $1,2 \mathrm{a}$ & $0,9 \mathrm{a}$ & 2,07 & 157,0 \\
\hline Total & $95,9 \mathrm{a}$ & $73,4 \mathrm{a}$ & $94,6 \mathrm{a}$ & $59,6 \mathrm{~b}$ & $99,7 \mathrm{a}$ & 11,32 & 36,2 \\
\hline \multicolumn{8}{|l|}{ Carabidae } \\
\hline Abaris sp. & $0,2 \mathrm{a}$ & $0,3 \mathrm{a}$ & $0,3 \mathrm{a}$ & $0,2 \mathrm{a}$ & $0,3 \mathrm{a}$ & 0,17 & 68,9 \\
\hline Callida scutellaris & $0,1 \mathrm{a}$ & $0,1 \mathrm{a}$ & $0,1 \mathrm{a}$ & $0,1 \mathrm{a}$ & $0,1 \mathrm{a}$ & 0,05 & 115,7 \\
\hline Metius sp. & $0,2 \mathrm{a}$ & $0,2 \mathrm{a}$ & $0,3 \mathrm{a}$ & $0,2 \mathrm{a}$ & $0,2 \mathrm{a}$ & 0,07 & 72,3 \\
\hline Total & $0,7 \mathrm{a}$ & $0,6 \mathrm{a}$ & $0,8 \mathrm{a}$ & $0,5 \mathrm{a}$ & $0,7 \mathrm{a}$ & 0,18 & 56,1 \\
\hline Cicindelidae & $0,01 \mathrm{a}$ & $0,01 \mathrm{a}$ & $0,01 \mathrm{a}$ & $0,01 \mathrm{a}$ & $0,01 \mathrm{a}$ & 0,09 & 257,1 \\
\hline Staphylinidae & $0,01 \mathrm{a}$ & $0,1 \mathrm{a}$ & $0,1 \mathrm{a}$ & $0,01 \mathrm{a}$ & $0,01 \mathrm{a}$ & 0,06 & 280,3 \\
\hline Dermaptera & $0,03 \mathrm{a}$ & $0,01 \mathrm{a}$ & $0,01 \mathrm{a}$ & $0,05 \mathrm{a}$ & $0,01 \mathrm{a}$ & 0,03 & 191,4 \\
\hline \multicolumn{8}{|l|}{ Araneae } \\
\hline Lycosidae & $0,7 \mathrm{a}$ & $0,5 \mathrm{~b}$ & $0,7 \mathrm{a}$ & $0,6 \mathrm{a}$ & $0,6 \mathrm{a}$ & 0,11 & 34,9 \\
\hline Linyphiidae & $0,2 \mathrm{a}$ & $0,1 \mathrm{a}$ & $0,2 \mathrm{a}$ & $0,1 \mathrm{a}$ & $0,2 \mathrm{a}$ & 0,07 & 93,0 \\
\hline Salticidae & $0,3 \mathrm{a}$ & $0,3 \mathrm{a}$ & $0,4 \mathrm{a}$ & $0,2 \mathrm{a}$ & $0,2 \mathrm{a}$ & 0,11 & 80,1 \\
\hline Total & $2,5 \mathrm{a}$ & $1,8 \mathrm{~b}$ & $2,2 a$ & $1,9 \mathrm{a}$ & $2,4 a$ & 0,28 & 26,7 \\
\hline \multicolumn{8}{|l|}{ Fitófagos } \\
\hline Acrididae & $3,5 \mathrm{a}$ & $3,3 \mathrm{a}$ & $3,5 \mathrm{a}$ & $3,3 \mathrm{a}$ & $3,4 a$ & 0,53 & 32,2 \\
\hline Gryllidae & $1,5 \mathrm{a}$ & $1,9 \mathrm{a}$ & $1,5 \mathrm{a}$ & $1,8 \mathrm{a}$ & $1,7 \mathrm{a}$ & 0,32 & 37,9 \\
\hline
\end{tabular}

${ }^{(1)}$ Médias seguidas da mesma letra, na horizontal, entre sistemas de plantio e entre culturas, não diferem entre si pelo teste de Tukey a 5\% de probabilidade; não foram significativas as interações entre manejo do solo e culturas. 
Pteronemobius presentes em pastagem, enquanto Tennis (1983) constatou que a diminuição da densidade de várias espécies de grilos presentes em campos de plantas silvestres deveu-se à predação por aranhas. Neste estudo, aranhas, com destaque para espécies da família Lycosidae, ocorreram em número significativamente maior no plantio direto do que no convencional, nas duas safras (Tabelas 2 e 3 ).

Na consorciação soja-milho, espécies de Acrididae foram mais numerosas apenas na safra 1996/97, enquanto espécies pertencentes à família Gryllidae não se mostraram influenciadas pela diversificação de culturas (Tabelas 2 e 3). A falta de consistência de resultados quanto à Acrididae deveu-se, provavelmente, a fatores não controlados no experimento. De acordo com Tonhasca Junior (1994), algumas espécies de insetos são mais afetadas por variações nos padrões de condições climáticas, e, como citado anteriormente, a precipitação pluvial ocorreu com intensidade diferente nas duas safras estudadas.

Tabela 5. Número médio de artrópodes predadores e fitófagos amostrados com armadilhas de solo na entressafra/ 1998. Jaboticabal, $\mathrm{SP}^{(1)}$.

\begin{tabular}{|c|c|c|c|c|c|c|c|}
\hline \multirow[t]{2}{*}{ Categorias taxonômicas } & \multicolumn{2}{|c|}{ Manejo do solo } & \multicolumn{3}{|c|}{ Cultura utilizada na parcela } & \multirow[t]{2}{*}{ Erro-padrão } & \multirow[t]{2}{*}{$\mathrm{CV}(\%)$} \\
\hline & Direto & Convencional & Soja & Milho & Soja $\mathrm{x}$ milho & & \\
\hline \multicolumn{8}{|l|}{ Predadores } \\
\hline \multicolumn{8}{|l|}{ Formicidae } \\
\hline Brachymyrmex sp. & $3,2 \mathrm{a}$ & $0,8 \mathrm{a}$ & $1,3 \mathrm{a}$ & $1,8 \mathrm{a}$ & $2,9 \mathrm{a}$ & 1,59 & 159,2 \\
\hline Camponotus spp. & $1,9 \mathrm{a}$ & $0,5 b$ & $1,5 \mathrm{a}$ & $1,0 \mathrm{a}$ & $1,0 \mathrm{a}$ & 0,54 & 93,5 \\
\hline Crematogaster sp. & $9,8 \mathrm{a}$ & $0,1 \mathrm{a}$ & $0,8 \mathrm{a}$ & $13,7 \mathrm{a}$ & $0,1 \mathrm{a}$ & 11,23 & 260,1 \\
\hline Dorymyrmex sp. & $5,5 b$ & $19,8 \mathrm{a}$ & $12,3 a$ & $15,7 \mathrm{a}$ & $9,9 \mathrm{a}$ & 7,10 & 95,6 \\
\hline Ectatomma sp. & $1,2 \mathrm{a}$ & $0,5 b$ & $1,2 \mathrm{a}$ & $0,5 \mathrm{a}$ & $0,8 \mathrm{a}$ & 0,36 & 90,4 \\
\hline Gnamptogenys sp. & $0,2 \mathrm{a}$ & $0,4 \mathrm{a}$ & $0,3 a$ & $0,1 \mathrm{a}$ & $0,4 a$ & 0,12 & 89,6 \\
\hline Odontomachus sp. & $0,1 \mathrm{a}$ & $0,0 \mathrm{a}$ & $0,0 \mathrm{a}$ & $0,1 \mathrm{a}$ & $0,1 \mathrm{a}$ & 0,06 & 163,0 \\
\hline Pheidole spp. & $37,8 \mathrm{a}$ & $44,8 \mathrm{a}$ & $42,6 a$ & $37,4 \mathrm{a}$ & $43,8 \mathrm{a}$ & 8,64 & 39,4 \\
\hline Solenopsis spp. & $2,1 \mathrm{a}$ & $4,7 \mathrm{a}$ & $6,6 \mathrm{a}$ & $0,4 \mathrm{a}$ & $3,3 \mathrm{a}$ & 4,46 & 261,6 \\
\hline Total & $61,8 \mathrm{a}$ & $71,3 \mathrm{a}$ & $66,7 \mathrm{a}$ & $70,7 \mathrm{a}$ & $66,2 \mathrm{a}$ & 16,94 & 50,8 \\
\hline Carabidae & $0,2 \mathrm{a}$ & $0,1 \mathrm{a}$ & $0,2 \mathrm{a}$ & $0,1 \mathrm{a}$ & $0,1 \mathrm{a}$ & 0,12 & 103,6 \\
\hline \multicolumn{8}{|l|}{ Araneae } \\
\hline Lycosidae & $0,5 \mathrm{a}$ & $0,5 \mathrm{a}$ & $0,6 \mathrm{a}$ & $0,5 \mathrm{a}$ & $0,4 a$ & 0,15 & 62,9 \\
\hline Linyphiidae & $0,5 \mathrm{a}$ & $0,5 \mathrm{a}$ & $0,4 \mathrm{~b}$ & $0,5 b$ & $0,7 \mathrm{a}$ & 0,10 & 41,7 \\
\hline Salticidae & $0,3 \mathrm{a}$ & $0,2 \mathrm{a}$ & $0,3 \mathrm{a}$ & $0,2 \mathrm{a}$ & $0,2 \mathrm{a}$ & 0,12 & 99,1 \\
\hline Total & $2,1 \mathrm{a}$ & $1,9 \mathrm{a}$ & $2,1 \mathrm{a}$ & $2,2 \mathrm{a}$ & $1,7 \mathrm{a}$ & 0,34 & 32,2 \\
\hline \multicolumn{8}{|l|}{ Fitófagos } \\
\hline Acrididae & $3,7 \mathrm{a}$ & $3,4 \mathrm{a}$ & $3,4 a$ & $3,7 \mathrm{a}$ & $3,6 \mathrm{a}$ & 0,54 & 29,9 \\
\hline Gryllidae & $1,7 \mathrm{a}$ & $1,8 \mathrm{a}$ & $1,7 \mathrm{a}$ & $1,7 \mathrm{a}$ & $1,9 a$ & 0,38 & 43,7 \\
\hline
\end{tabular}

${ }^{(1)}$ Médias seguidas da mesma letra, na horizontal, entre sistemas de plantio e entre culturas, não diferem entre si pelo teste de Tukey a $5 \%$ de probabilidade; não foram significativas as interações entre manejo do solo e cultura. 


\section{Conclusões}

1. Sistemas de plantio direto da soja e do milho aumentam o número de aranhas, carabídeos e formigas.

2. A presença do milho favorece a ocorrência de insetos da ordem Dermaptera em sistemas de consorciação soja-milho.

\section{Agradecimentos}

Ao Dr. José Carlos Barbosa, FCAV-Unesp, pelo auxílio nas análises estatísticas; à Dra Isabela Maria P. Rinaldi, do Instituto de Biociências/Unesp, Botucatu, SP, pela identificação das aranhas; aos Drs. Carlos Roberto F. Brandão e Carlos Campaner, do Museu de Zoologia/USP, São Paulo, SP, pela identificação das formigas e carabídeos, respectivamente; ao Sr. Jairo Guimarães Figueiredo, FCAVUnesp, pela colaboração na execução dos experimentos em campo.

\section{Referências}

ANDERSEN, A. Plant protection in spring cereal production with reduced tillage. II. Pests and beneficial insects. Crop Protection, Oxford, v. 18, p. 651-657, 1999.

ANDOW, D. A. Fate of eggs of first-generation Ostrinia nubilalis (Lepidoptera: Pyralidae) in three conservation tillage systems. Environmental Entomology, Lanham, v. 21 , p. $388-393,1992$.

BOOIJ, C. J. H.; NOORLANDER, J. Farming systems and insect predators. Agriculture, Ecosystems and Environment, Amsterdam, v. 40, p. 125-135, 1992.

BRUST, G. E. A method for observing below-ground pestpredator interactions in corn agroecosystems. Journal of Entomological Science, Griffin, v. 26, p. 1-8, 1991.

BRUST, G. E.; HOUSE, G. J. Effects of soil moisture, notillage and predators on Southern corn rootworm (Diabrotica undecimpunctata howardi) survival in corn agroecosystem. Agriculture, Ecosystems and Environment, Amsterdam, v. 31, p. 199-215, 1990.

CÁRCAMO, H. A.; SPENCE, J. R. Crop type effects on the activity and distribution of ground beetle (Coleoptera: Carabidae). Environmental Entomology, Lanham, v. 23, p. 684-692, 1994.
CLARK, M. S.; GAGE, S. H.; SPENCE, J. R. Habitats and management associated with common ground beetles (Coleoptera: Carabidae) in a Michigan agricultural landscape. Environmental Entomology, Lanham, v. 26, p. 519-527, 1997.

CLARK, M. S.; LUNA, J. M.; STONE, N. D.; YOUNGMAN, R. R. Generalist predator consumption of armyworm (Lepidoptera: Noctuidae) and effect of predator removal on damage in no-till corn. Environmental Entomology, Lanham, v. 23, p. 617-622, 1994.

EMDEN, H. F. van; WILLIAMS, G. F. Insect stability and diversity in agro-ecosystems. Annual Review of Entomology, Palo Alto, v. 19, p. 455-474, 1974.

FRENCH, B. W.; ELLIOTT, N. C.; BERBERET, R. C. Reverting conservation reserve program lands to wheat and livestock production: effects on ground beetles (Coleoptera: Carabidae) assemblages. Environmental Entomology, Lanham, v. 27, p. 1323-1335, 1998.

GARCIA, M. A.; ALTIERI, M. A. Explaining differences in flea beetle Phyllotreta cruciferae Goeze densities in simple and mixed broccoli cropping systems as a function of individual behavior. Entomologia Experimentalis et Applicata, Dordrecht, v. 62, p. 201-209, 1992.

HOOK, R. I. van. Energy and nutrient dynamics of spider and orthopteran populations in a grassland ecosystem. Ecological Monographs, Washington, v. 41, p. 1-26, 1971.

LETOURNEAU, D. K. Two examples of natural enemy augmentation: a consequence of crop diversification. In: GLIESSMAN, S. R. (Ed.). Agroecology, researching the ecological basis for sustainable agriculture. New York: Springer, 1990. p. 11-29.

MARASAS, M. E.; SARANDON, S. J.; CICCHINO, A. C. Efecto de la labranza sobre la coleóptero fauna edáfica en un cultivo de trigo en la provincia de Buenos Aires (Argentina). Ciencia del Suelo, Buenos Aires, v. 15, p. 59-63, 1997.

SKOVGARD, H.; PATS, P. Effects of intercropping on maize stemborers and their natural enemies. Bulletin of Entomological Research, Wallingford, v. 86, p. 599-607, 1996.

STINNER, B. R.; HOUSE, G. J. Arthropods and other invertebrates in conservation-tillage agriculture. Annual Review of Entomology, Palo Alto, v. 35, p. 299-318, 1990. 
SUNDERLAND, K. D.; LÖVEI, G. L.; FENLON, J. Diets and reproductive phenologies of the introduced ground beetles Harpalus affinis and Clivina australasiae (Coleoptera: Carabidae) in New Zealand. Australian Journal of Zoology, Collingwood, v. 43, p. 39-50, 1995.

SYMONDSON, W. O. C.; GLEN, D. M.; WILTSHIRE, C. W.; LANGDON, C. J.; LIDDELL, J. E. Effects of cultivation techniques and methods of straw disposal on predation by Pterostichus melanarius (Coleoptera: Carabidae) upon slugs (Gastropoda: Pulmonata) in an arable field. Journal of Applied Ecology, Oxford, v. 33, p. 741-753, 1996.

TENNIS, P. Survivorship, spatial pattern, and habitat structure of field crickets (Orthoptera: Gryllidae) in two old fields. Environmental Entomology, Lanham, v. 12, p. 110-116, 1983.
TONHASCA JUNIOR, A. Effects of agroecosystem diversification on natural enemies of soybean herbivores. Entomologia Experimentalis et Applicata, Dordrecht, v. 69 , p. 83-90, 1993.

TONHASCA JUNIOR, A. Response of soybean herbivores to two agronomic practices increasing agroecosystem diversity. Agriculture, Ecosystems and Environment, Amsterdam, v. 48, p. 57-65, 1994.

TONHASCA JUNIOR, A.; STINNER, B. R. Effects of strip intercropping and no-tillage on some pests and beneficial invertebrates of corn in Ohio. Environmental Entomology, Lanham, v. 20, p. 1251-1258, 1991.

WATMOUGH, R. H.; KFIR, R. Predation on pupae of Helicoverpa armigera Hbn. (Lep., Noctuidae) and its relation to stem borer numbers in summer grain crops. Journal of Applied Entomology, Berlin, v. 119, n. 10, p. $679-688,1995$. 